\title{
Primary Fallopian Tube Adenocarcinoma Discovered with the Vaginal Cytology: A Case Report with Pathological and Immunohistochemical Investigation
}

\author{
Tomoko Honda1 ${ }^{1}$, Yuka Hiraku ${ }^{2}$, Ryogo Aoki ${ }^{1}$, Kenji Niwa ${ }^{3}$, Naoki Watanabe ${ }^{1}$, Takuji Tanaka ${ }^{*}$ \\ ${ }^{1}$ Department of Diagnostic Pathology (DDP) \& Research Center of Diagnostic Pathology (RC-DiP), Gifu Municipal Hospital, Gifu \\ City, Japan \\ ${ }^{2}$ Department of Obstetrics \& Gynecology, Gifu Municipal Hospital, Gifu City, Japan \\ ${ }^{3}$ Department of Obstetrics \& Gynecology, Gujo City Hospital, Gujo City, Japan \\ Email: *tmntt08@gmail.com, ${ }^{*}$ takutt@gmhosp.gifu.gifu.jp
}

How to cite this paper: Honda, T., Hiraku, Y., Aoki, R., Niwa, K., Watanabe, N. and Tanaka, T. (2018) Primary Fallopian Tube Adenocarcinoma Discovered with the Vaginal Cytology: A Case Report with Pathological and Immunohistochemical Investigation. Open Journal of Pathology, 8, 85-93.

https://doi.org/10.4236/ojpathology.2018.8

$\underline{3010}$

Received: June 11, 2018

Accepted: July 10, 2018

Published: July 13, 2018

Copyright (๑) 2018 by authors and Scientific Research Publishing Inc. This work is licensed under the Creative Commons Attribution International License (CC BY 4.0).

http://creativecommons.org/licenses/by/4.0/ (c) (i) Open Access

\begin{abstract}
Background: Primary fallopian tube cancer is an extremely rare gynecological malignancy. Aim: To discuss, through a case report, the diagnostic process by means of cytology immunohistochemistry. Case Presentation: A 47-year-old Japanese woman, who also had lung cancer, presented with enlarged para-aortic lymph node without any symptoms. Based on the vaginal cytology report suggestive of gynecologic malignant tumor (possibly fallopian tube adenocarcinoma), primary surgery comprised of total abdominal hysterectomy and bilateral salpingo-oophorectomy was performed. Histopathology and immunohistochemistry examinations revealed primary fallopian tube carcinoma with metastasis of para-aortic lymph node. She is free from recurrence and metastases 9 months after the surgery and chemotherapy. Conclusion: Although primary fallopian tube cancer is a rare gynecologic malignancy, vaginal cytology may be useful for detecting fallopian tube carcinoma.
\end{abstract}

\section{Keywords}

Primary Fallopian Tube Carcinoma, Vaginal Cytology, Histopathology, Immunohistochemistry

\section{Introduction}

Primary fallopian tube cancer is extremely rare, accounting for $0.14 \%-1.8 \%$ of malignancies in women annually [1] [2] [3] [4]. Although it is hard to differen- 
tiate histopathologically and clinically from ovarian cancer, the management of both is similar [4]. The etiology of this malignancy is unknown. However, fallopian tube cancer has been reported in high-risk breast-ovarian cancer families with germ-line mutations of BRCA-1 and BRCA-2 [4]. The CA-125 antigen is often expressed by this malignancy, and serum level of CA-125 is elevated in $>80 \%$ of patients with this disease pretreatment [4].

Several case reports described usefulness of cervical or vaginal smears for the diagnosis of fallopian tube cancer. However, positive Papanicolaou (Pap)-stained smears have been reported in only $0 \%-2.3 \%$ of cases [4]. The diagnosis of this malignancy is usually first made by a pathologist on histopathological examinations. Since it is difficult to differentiate fallopian tubal cancer from ovarian cancer, the patients with at least one of the following criteria [5] should be diagnosed as this malignancy: 1) the main tumor is in the tube and develops from the endosalpinx; 2) the pattern histopathologically reproduces the tubal epithelium and often shows a papillary pattern; 3 ) if the wall is involved, the transition between normal and malignant epithelium should be observed; and 4) the ovaries and endometrium are either normal or contain less tumor than the tube.

We report here a case of the right fallopian tube cancer, which was discovered by the vaginal smear during the close inspection of para-aortic adenopathy found when general searching metastasis of the right upper lobe lung cancer. Although the tubal cancer was very small and the patient had no significant clinical signs, we noticed its metastasis to the para-aortic lymph node.

\section{Case Presentation}

This case report presents a 47-year-old Japanese female housewife. She was nulliparous and had no previous known gynecological problems. Her past history included removal of thyroid tumor at 43 years old. In August, 2017, her medical checkup revealed lung cancer (well-differentiated bronchioloalveolar carcinoma) of the right upper lobe (Figure 1). A week later, positron emission tomography (PET)-computed tomography (CT) examination revealed abnormal 18-fluorodeoxyglucose (FDG) uptake in the para-aortic lymph node, suggesting lymph node metastasis of lung cancer. Endoscopic ultrasound-guided fine needle aspiration (EUS-FNA) examination of the lymph node confirmed poorly-differentiated adenocarcinoma on the Pap-stained cytologic (Figure 2(A)) and on the $\mathrm{H} \&$ E-stained histopathologic specimens (Figure 2(B)). However, this metastatic lesion was histopathologically different from lung cancer. Therefore, we suspected the metastatic lesion was derived from malignancy in organ other than lung. One month later, her vaginal smear was positive for adenocarcinoma (Figure 3(A)), although the pelvic contrast magnetic resonance imaging (MRI) examination did not show any abnormalities in the uterine corpus, uterine cervix, and bilateral adnexa. Cervical Pap smear reported CIN1 with koilocytosis. In order to determine the primary site of the lymph node malignancy, we tried to diagnose endometrial cytology and histology, but we were not able to 


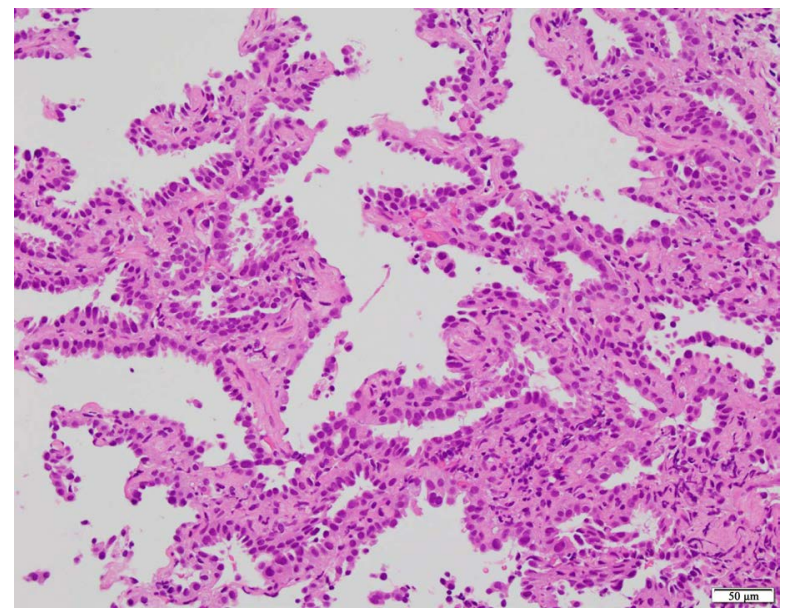

Figure 1. Biopsy specimens of lung tumor in the right upper lobe show bronchioloalveolar carcinoma, so-called BAC. H \& E-stain, bar $=50 \mu \mathrm{m}$.
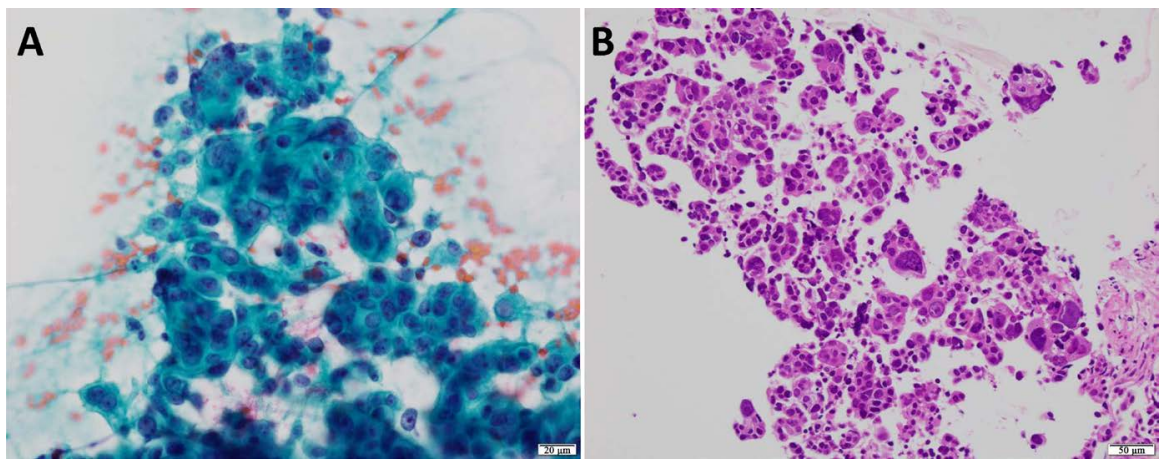

Figure 2. EUS-FNA specimens from para-aortic lymph node shows papillar adenocarcinoma on (A) the Papanicolaou-stained smears and (B) H \& E-stained histological section. Bars $=(A) 20 \mu \mathrm{m}$ and (B) $50 \mu \mathrm{m}$.

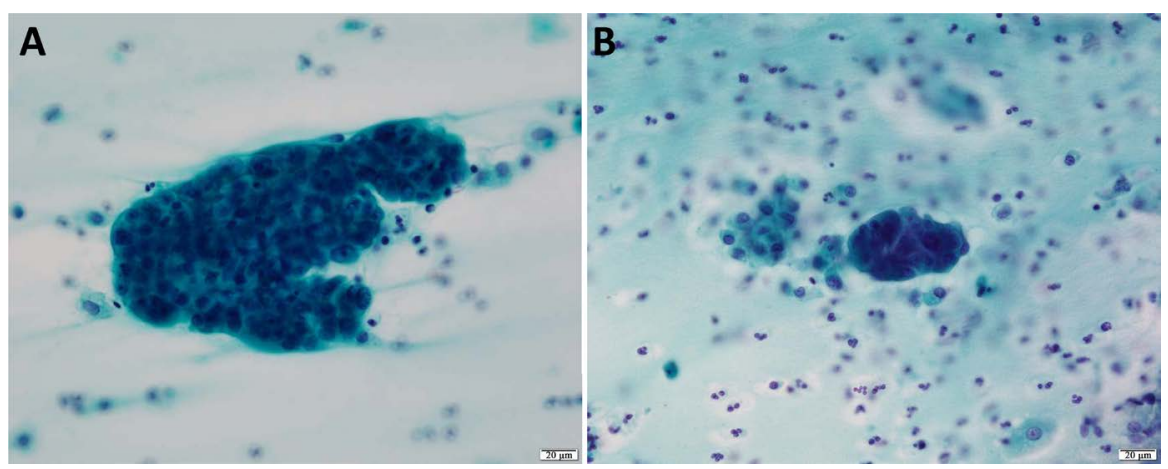

Figure 3. The $1^{\text {st }}$ vaginal smear shows papillary adenocarcinoma (A). Papillary clusters of atypical glandular cells with increased nucleo-cytoplasmic ratio, hyperchromatic nuclei, and large nucleoli. On the $2^{\text {nd }}$ vaginal smear (B), only one papillary cluster consisted of atypical glandular cells with enlarged nuclei and nucleoli, suggesting adenocarcinoma. (A) and (B), Papanicolaou stain, bars $=20 \mu \mathrm{m}$.

obtain samples because cervical duct was closed down. Serum levels of tumor markers, including CA-125 (10.7 U/ml, reference value: $~ 35 \mathrm{U} / \mathrm{ml})$, CA19-9 $(<2.0 \mathrm{U} / \mathrm{ml}$, reference value: $\sim 37 \mathrm{U} / \mathrm{ml})$, SCC $(0.6 \mathrm{ng} / \mathrm{ml}$, reference value: $\sim 1.5$ 
$\mathrm{ng} / \mathrm{ml}$ )were normal range. Re-examination of vaginal smear on October 11, 2017 was also positive for adenocarcinoma (Figure $3(\mathrm{~B})$ ), while cervical curettage showed no abnormality. Gastrointestinal tract studies by upper and lower endoscopy showed normal. Although primary site of the malignancy was unknown, we suspected gynecological malignancy, possibly fallopian tube cancer. We decided then to perform abdominal simple hysterectomy, bilateral appendages extirpation and removal of para-aortic lymph nodes in November, 2017. During the operation, peritoneal lavage cytology was positive for adenocarcinoma (Figure 4), but the peritoneum was macroscopically normal. While the uterus, left ovary, the left fallopian tube, and the right ovary showed no abnormalities (Figure 5(A)), the right fallopian tube (Figure 5(A), circled) was slightly enlarged and nodular. The nodular lesions were consisted of diffusely proliferated tumor cells with small and large round nuclei and prominent nucleoli, suggesting poorly differentiated adenocarcinoma (Figure 5(B)). Immunohistochemistry revealed that cancer cells were positive against AE1/AE3, CK7, and ER, while they were negative for CK20, CEA, and calretinin. Most cancer cells showed positive reaction against p53 (Figure 5(C)). A few tubal epithelial cells also had nuclei slightly positive for p53. Similar cancer cells were detected in the para-aortic lymph node. Our final diagnosis was right fallopian tube adenocarcinoma (poorly differentiated) with para-aortic lymph node metastasis (Stage IIIc: pT1cN1M0). Four months later, the upper lobectomy (Figure 6(A)) of the right lung was performed and the lung cancer was histopathogically well-differentiated adenocarcinoma (Stage IB, T2aN0M0) (Figure 6(B)). She received four courses of systemic chemotherapy with paclitaxel $\left(175 \mathrm{mg} / \mathrm{m}^{2}\right)$ and carboplatin (AUC: $6 \mathrm{mg} / \mathrm{ml} / \mathrm{min}$ every 21 days) combined with bevacizumab (15 mg/kg).

The patient is doing fine at 9-months follow-up with no evidence of reccurrence/distant metastasis of two malignancies after surgery and chemotherapy. We should intensively follow-up, since the actuarial 5-year survival rate was $38 \%-50 \%$ [6] [7].

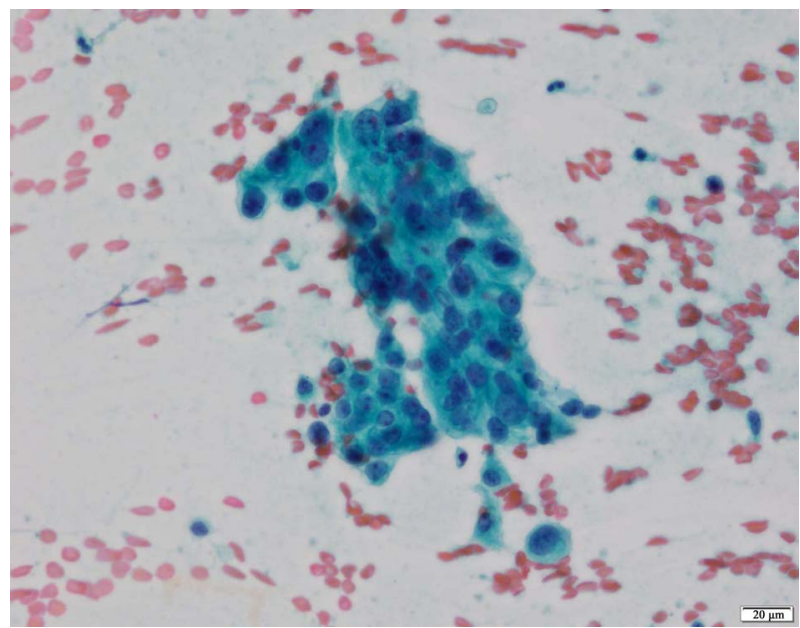

Figure 4. Peritoneal lavage cytology was suggestive of adenocarcinoma. The sheet of atypical glandular cells with large nucleoli is present. Papanicolaou stain, bar $=20 \mu \mathrm{m}$. 

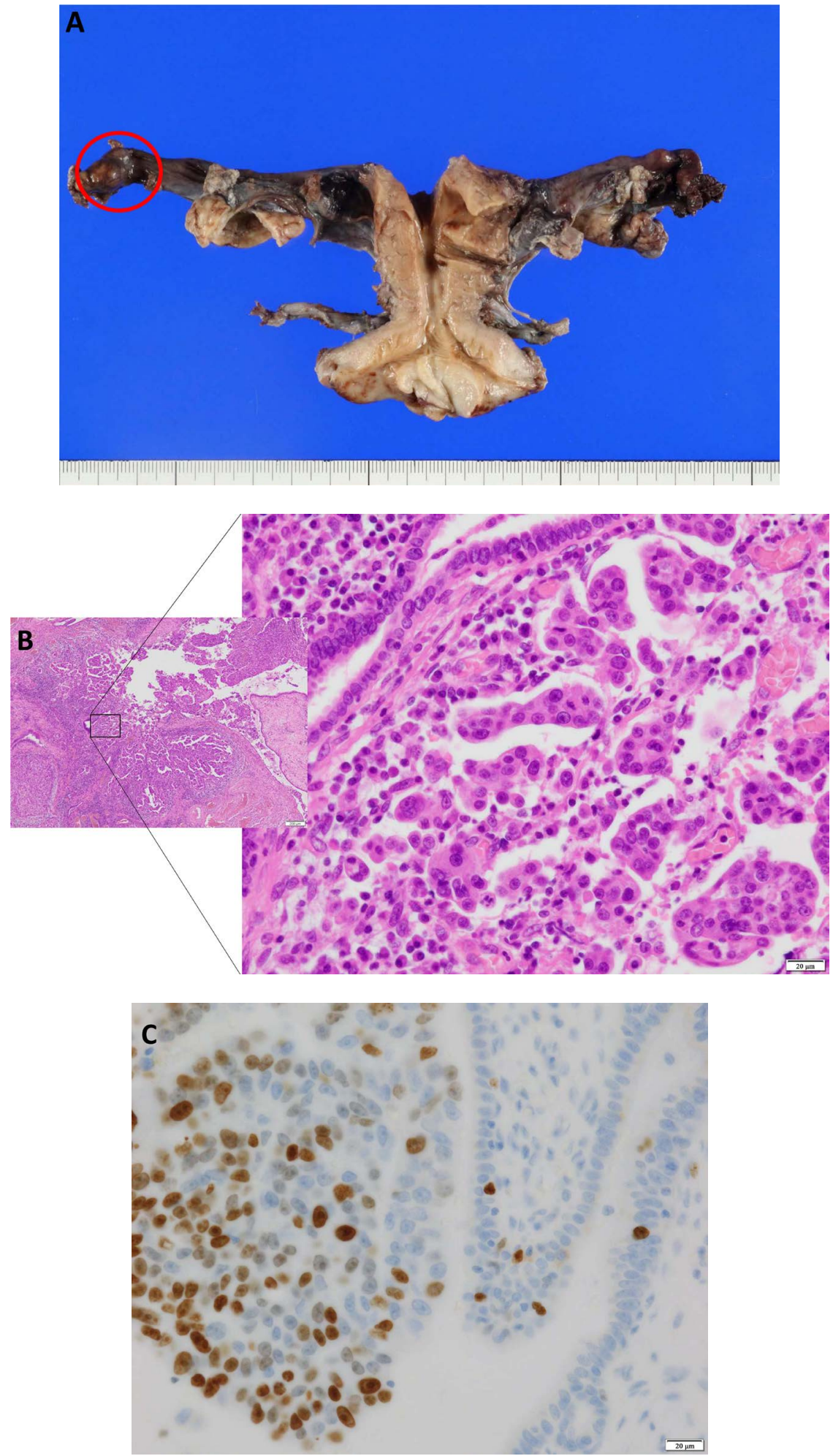

Figure 5. (A) Macroscopic view of the resected uterus and bilateral appendages. Note: the right tube (circled) was slightly enlarged. (B) Histological examination reveals that the tumor is papillary adenocarcinoma. (C) p53-immunohistochemistry shows that most of cancer cells have nuclear positive reaction against p53. Note: a few normal tubal epithelial cells are also positive for p53 in their nucleus. (B) $\mathrm{H} \& \mathrm{E}$ stain, bars $=200 \mu \mathrm{m}$ (low magnification) and 20 $\mu \mathrm{m}$ (high magnification) and (C) p53 immunohistochemistry, bar $=20 \mu \mathrm{m}$. 


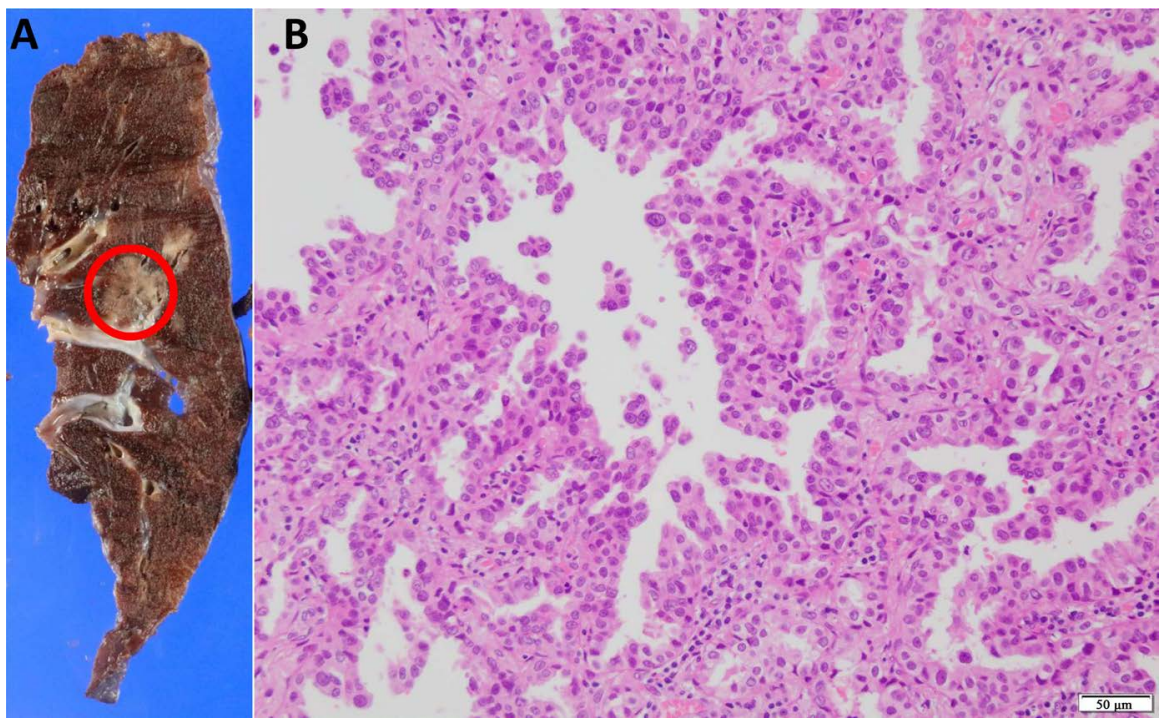

Figure 6. Macroscopic view of (A) lung tumor in the right upper lobe. Histopathologically, the tumor was (B) papillary adenocarcinoma (papillary $40 \%$, acinar $20 \%$, and lepidic $40 \%$ ) without metastases. (B) $\mathrm{H} \& \mathrm{E}$ stain, bar $=50 \mu \mathrm{m}$.

\section{Discussion}

Primary fallopian tube carcinoma is very rare with an average of $0.3 \%$ of all the gynecological malignancies [8], although its exact frequency may be underestimated because it has the same pathological features as serous epithelial ovarian cancer or primary peritoneal serous carcinoma [9]. Tubal carcinomas commonly develop in postmenopausal women in their fifth or sixth decades. Most of the patients are asymptomatic or they tend to present with certain non-specific symptoms. In most of these patients, infertility and chronic pelvic disease are commonly associated. Because the lumen of fallopian tubes anatomically continues with uterine cavity, cancer cells developed in the fallopian tube could easily reach to uterine cavity, when compared to ovarian cancer. Therefore, endometrial Pap smears show positive results at early stage of tubal cancer [10]. In the present case, we could not perform endometrial Pap smears because of closed cervical duct. However, based on the positive results vaginal smears, we suspected gynecological malignancy, possibly fallopian tube cancer, and did abdominal simple hysterectomy and bilateral appendages extirpation with removal of para-aortic lymph nodes. As a result, we detected the right tubal adenocarcinoma. Our findings suggest that in addition to endometrial and cervical cytology, vaginal cytology is useful for early detection of fallopian tube cancer [10].

When compared to endometrial cancer, adenocarcinoma cells originated from fallopian tube on the endometrial Pap smears have been reported to show several characteristics: 1) background is clear or clean; 2) number of atypical cells is small; 3) clusters of atypical cells are papillary or small spherical pattern; 4) atypical cell are frequently degenerated; and 5) neutrophils in the cytoplasm of atypical cells are infrequent [11]. In our case, cancer cells were found as papillary clusters with clear background on the vaginal smear. Cytological specimens ob- 
tained by hydrotubation were reported to be useful for diagnosis adenocarcinoma in situ in the fallopian tube [12]. Chen et al. [13] have recently developed a standard procedure to collect fallopian tube brushing from fresh surgical specimens. They have proved that tubal brushing cytology is useful for early detection of serous tubal intraepithelial carcinoma (STIC).

Primary fallopian tube carcinoma is rarely suspected preoperatively and resembles histologically and clinically primary ovarian carcinoma [6]. The diagnostic criteria for primary fallopian tube cancer, which were proposed by $\mathrm{Hu}$ et al. [5], were revised by Sedis [3] [7]. They include: 1) main tumor should develop in the fallopian tube; 2) the histological pattern reproduces the epithelium of the tubal epithelium; 3) transition from benign to malignant tubal epithelium is demonstrable; and 4) ovaries or endometrium are either normal or contain a tumor that is smaller than the tumor in the tube. More than $90 \%$ of primary fallopian tube carcinoma was papillary adenocarcinoma [9]. Histopathology of our case satisfied these two criteria.

Based on these two criteria, the fallopian tube cancer has been considered to be extremely rare at $0.5 \%-03 \%$ of all gynecological neoplasms [14]. However, the concept that serous tubal intraepithelial carcinoma (STIC) is a precursor lesion of high grade serous adenocarcinoma (HGSA) has recently been proposed [15]. As presented, vaginal cytology was useful for detecting tubal adenocarcinoma. If STIC is a precursor lesion of HGSA, endometrial and cervical cytological diagnosis may show an important role in early diagnosis of HGSC [16]. We have recently experienced a case of STIC cytologically diagnosed during the operation [17].

Immunohistochemical expression of p53 in the tubal epithelium is known to be an early biomarker of tubal malignancy [4] [18] [19]. In our case, most of adenocarcinoma cells in the right tube were immunohistochemically positive for p53. Interestingly, normal-looking tubal epithelial cells were also positive for p53. These findings may suggest that histopathologically normal tubal epithelium already has genetic alteration.

\section{Acknowledgements}

We thank the editor and reviewers for the constructive comments, which helped us to improve the manuscript. This case report was approved by the patient verbal consent.

\section{Competing Interest}

The authors declare that they have no competing interest.

\section{Authors' Contributions}

\begin{tabular}{ccccccc}
\hline Authors' contributions & TH & YH & RA & KN & NW & TT \\
\hline Research concept and design & $\checkmark$ & & & & & $\checkmark$ \\
Collection and/or assembly of data & $\checkmark$ & $\checkmark$ & $\checkmark$ & $\checkmark$ & $\checkmark$ & $\checkmark$ \\
\hline
\end{tabular}




\section{Continued}

\begin{tabular}{|c|c|c|c|c|c|c|}
\hline Immunohistochemistry & $\checkmark$ & & $\checkmark$ & & & \\
\hline Data analysis and interpretation & $\checkmark$ & $\checkmark$ & $\checkmark$ & $\checkmark$ & $\checkmark$ & $\checkmark$ \\
\hline Writing the article & $\checkmark$ & & & & & $\checkmark$ \\
\hline Critical revision of the article & $\checkmark$ & $\checkmark$ & & & & $\checkmark$ \\
\hline Critical revision of the article & $\checkmark$ & $\checkmark$ & $\checkmark$ & $\checkmark$ & $\checkmark$ & $\checkmark$ \\
\hline
\end{tabular}

\section{References}

[1] Baekelandt, M., Jorunn Nesbakken, A., Kristensen, G.B., Trope, C.G. and Abeler, V.M. (2000) Carcinoma of the Fallopian Tube. Cancer, 89, 2076-2084. https://doi.org/10.1002/1097-0142(20001115)89:10<2076::AID-CNCR7>3.0.CO;2-V

[2] Eddy, G.L., Copeland, L.J., Gershenson, D.M., Atkinson, E.N., Wharton, J.T. and Rutledge, F.N. (1984) Fallopian Tube Carcinoma. Obstetrics \& Gynecology, 64, 546-552.

[3] Sedlis, A. (1961) Primary Carcinoma of the Fallopian Tube. Obstetrical \& Gynecological Survey, 16, 209-226.

[4] Pectasides, D., Pectasides, E. and Economopoulos, T. (2006) Fallopian Tube Carcinoma: A Review. Oncologist, 11, 902-912. https://doi.org/10.1634/theoncologist.11-8-902

[5] Hu, C.Y., Taymor, M.L. and Hertig, A.T. (1950) Primary Carcinoma of the Fallopian Tube. American Journal of Obstetrics \& Gynecology, 59, 58-67. https://doi.org/10.1016/0002-9378(50)90341-3

[6] Piura, B. and Rabinovich, A. (2000) Primary Carcinoma of the Fallopian Tube: Study of 11 Cases. European Journal of Obstetrics \& Gynecology and Reproductive Biology, 91, 169-175. https://doi.org/10.1016/S0301-2115(99)00264-X

[7] Sedlis, A. (1978) Carcinoma of the Fallopian Tube. Surgical Clinics of North America, 58, 121-129. https://doi.org/10.1016/S0039-6109(16)41439-8

[8] Ng, P. and Lawton, F. (1998) Fallopian Tube Carcinoma-A Review. Annals of the Academy of Medicine. Singapore, 27, 693-697.

[9] Kalampokas, E., Kalampokas, T. and Tourountous, I. (2013) Primary Fallopian Tube Carcinoma. European Journal of Obstetrics \& Gynecology and Reproductive Biology, 169, 155-161. https://doi.org/10.1016/j.ejogrb.2013.03.023

[10] Kajiwara, H., Yasuda, M., Itoh, H., Muramatsu, T., Hirasawa, T., Miyamoto, T., Shinozuka, T. and Osamura, Y. (1997) A Case of Double Cancers, Endocervical Adenocarcinoma and Tubal Adenocarcinoma, Which Were Found by Cytological Study in a Medical Checkup (in Japanese). The Journal of the Japanese Society of Clinical Cytology, 36, 633-635. https://doi.org/10.5795/jjscc.36.633

[11] Sasaki, T., Sakuragi, N., Ebina, Y., Shimizu, M., Arakawa, M. and Fujimoto, S. (2001) In Situ Carcinoma of the Fallopian Tube Detected by Positive Endometrial Smear: A Case Report. The Journal of the Japanese Society of Clinical Cytology, 40, 471-475. https://doi.org/10.5795/jjscc.40.471

[12] Takahashi, M., Kigawa, J., Ishihara, K., Shimada, M., Kamei, T. and Terakawa, N. (2002) Hydrotubation for Diagnosing Carcinoma in Situ of the Fallopian Tube: A Case Report. Acta Cytologica, 46, 735-737. https://doi.org/10.1159/000326986

[13] Chen, H., Klein, R., Arnold, S., Chambers, S. and Zheng, W. (2016) Cytologic Studies of the Fallopian Tube in Patients Undergoing Salpingo-Oophorectomy. Cancer Cell International, 16, 78. 
[14] Crum, C.P., Parkash, V., Alvarado-Cabrero, I., Piek, J.M.J., Bijron, J.G., Shaw, P., Carcangiu, M.L., Soslow, R., Ferry, J.A., van Diest, P.J. and Vang, R. (2014) Epithelial Tumours and Cysts. In: Kurman, R.J., Carcangiu, M.L., Herrington, C.S. and Young, R.H., Eds., WHO Classification of Tumours of Female Reproductive Organs, International Agency for Research on Cancer (IARC), Lyon, 103-112.

[15] Li, H.X., Lu, Z.H., Shen, K., Cheng, W.J., Malpica, A., Zhang, J., Wei, J.J., Zhang, Z.H. and Liu, J. (2014) Advances in Serous Tubal Intraepithelial Carcinoma: Correlation with High Grade Serous Carcinoma and Ovarian Carcinogenesis. International Journal of Clinical and Experimental Pathology, 7, 848-857.

[16] Lin, S.F., Gerry, E. and Shih, I.M. (2017) Tubal Origin of Ovarian Cancer-The Double-Edged Sword of Haemoglobin. The Journal of Pathology, 242, 3-6. https://doi.org/10.1002/path.4875

[17] Niwa, K., Ishuihara, T., Ueda, Y., Takenaka, M., Shiga, T., Mori, S., Kuwabara, K., Yamaguchi, Y. and Tanaka, T. (2018) A Case of Serous Tubal Intraepithelial Carcinoma Diagnosed by Cytology during the Operation. Open Journal of Pathology, 8 , 69-77.

[18] Mehra, K., Mehrad, M., Ning, G., Drapkin, R., McKeon, F.D., Xian, W. and Crum, C.P. (2011) STICS, SCOUTs and p53 Signatures; a New Language for Pelvic Serous Carcinogenesis. Frontiers in Bioscience, 3, 625-634.

[19] Piek, J.M., van Diest, P.J., Zweemer, R.P., Jansen, J.W., Poort-Keesom, R.J., Menko, F.H., Gille, J.J., Jongsma, A.P., Pals, G., Kenemans, P. and Verheijen, R.H. (2001) Dysplastic Changes in Prophylactically Removed Fallopian Tubes of Women Predisposed to Developing Ovarian Cancer. The Journal of Pathology, 195, 451-456. https://doi.org/10.1002/path.1000 